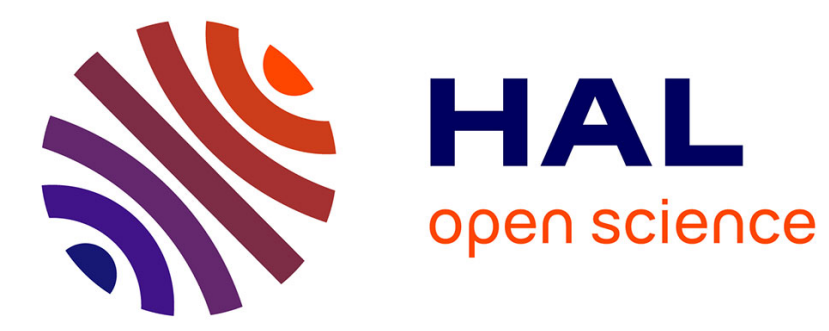

\title{
Lamb wave transducers built on periodically poled Z-cut LiNbO3 wafers
}

\author{
E. Courjon, N. Courjal, W. Daniau, G. Lengaigne, L. Gauthier-Manuel, S. \\ Ballandras, J. Hauden
}

\section{- To cite this version:}

E. Courjon, N. Courjal, W. Daniau, G. Lengaigne, L. Gauthier-Manuel, et al.. Lamb wave transducers built on periodically poled Z-cut LiNbO3 wafers. Journal of Applied Physics, 2007, 102 (11), pp.114107. 10.1063/1.2802566 . hal-00216090

\section{HAL Id: hal-00216090 \\ https://hal.science/hal-00216090}

Submitted on 28 Apr 2021

HAL is a multi-disciplinary open access archive for the deposit and dissemination of scientific research documents, whether they are published or not. The documents may come from teaching and research institutions in France or abroad, or from public or private research centers.
L'archive ouverte pluridisciplinaire HAL, est destinée au dépôt et à la diffusion de documents scientifiques de niveau recherche, publiés ou non, émanant des établissements d'enseignement et de recherche français ou étrangers, des laboratoires publics ou privés. 


\title{
Lamb wave transducers built on periodically poled Z-cut $\mathrm{LiNbO}_{3}$ wafers
}

\author{
E. Courjon, N. Courjal, W. Daniau, G. Lengaigne, L. Gauthier-Manuel, and S. Ballandras ${ }^{\text {a) }}$ \\ Institut FEMTO-ST, UMR 6174 CNRS-UFC-ENSMM-UTBM, F-25044 Besançon, France \\ J. Hauden \\ Photline Technologies, 16 Rue Jouchoux, F-25000 Besançon, France
}

(Received 24 November 2006; accepted 9 September 2007; published online 10 December 2007)

\begin{abstract}
In this paper, the development of piezoelectric transducers based on periodically poled ferroelectric domains is investigated. Optical quality $\mathrm{Z}$-cut $\mathrm{LiNbO}_{3}$ wafers have been used for the fabrication of test devices operating in the range of $10-100 \mathrm{MHz}$. The fabrication process is detailed and the characterization results are reported. A very good agreement between theoretical predictions achieved by periodic finite element analysis and experiments is observed, allowing for a precise identification of the excited modes. Elliptically polarized modes as well as quasipure longitudinal waves are pointed out. The robustness of the devices to lapping and polishing operations has also been tested. The possible utilizations of such transducers are discussed to conclude the paper.
\end{abstract} (C) 2007 American Institute of Physics. [DOI: 10.1063/1.2802566]

\section{INTRODUCTION}

The possibility to manufacture elastic waveguide based on a thin ferroelectric film deposited on a single crystal substrate and periodically poled to enable the excitation of surface acoustic waves has been demonstrated recently. ${ }^{1,2}$ The so-called piezoelectric interdigital transducer ${ }^{2}$ simply consists in a periodically poled ferroelectric layer grown on a conductive substrate. A metal coating is deposited atop this structure and used as an active electrode, the substrate being grounded, allowing for the excitation of the guided wave within the film. It has been successfully implemented and tested for the excitation of elliptically polarized waves in the frequency range of $1-3 \mathrm{GHz} .{ }^{2}$ Also in optics, ${ }^{3}$ periodically poled devices are widely used for signal processing applications. For instance, an extensive literature has been produced in that domain for the development of electro-optical and nonlinear devices for optical frequency converters and multipliers (see, for instance Ref. 4).

In this work, the possibility to develop periodically poled transducers (PPTs) on $\mathrm{LiNbO}_{3}$ is investigated, since this material is classically identified as well adapted for very high frequency applications. ${ }^{5}$ The first experiments have been performed on $500 \mu \mathrm{m}$ thick 3 in. Z-cut wafers of optical quality answering severe specifications on total thickness variation and side parallelism. ${ }^{6}$ The fabrication of PPTs on such wafers allows for the excitation of symmetrical Lamb modes with an operating frequency twice higher than those obtained using standard interdigital transducers. An insulating pattern is defined on one side of the wafer, enabling the implementation of a poling process using a liquid electrode. A poling sequence ${ }^{6}$ (maximum electric field of about $21 \mathrm{kV} \mathrm{mm}^{-1}$ ) was applied to fabricate 100 and $200 \mu \mathrm{m}$ period PPTs (50 and $100 \mu \mathrm{m}$ linewidths, respectively). The corresponding devices exhibiting, respectively, 500 and 400 periods have been successfully manufactured and tested and the measured electrical admittances compared to theoretical

${ }^{a)}$ Electronic mail: sylvain.ballandras@femto-st.fr predictions. The latter were obtained using our periodic finite element code ${ }^{7}$ providing a harmonic admittance when considering a synchronous excitation of all the PPT cells comparable to the admittance of our implemented (very long) PPTs. An excellent agreement is found between both results, enabling one to analyze almost all the peaks of the experimental setup response. Even low frequency parasitic signals were analyzed by the theoretical analysis. The $\mathrm{LiNbO}_{3}$ wafer then was lapped and polished to check the robustness of the PPTs to this kind of operation. The PPTs still operate after reducing the wafer thickness by a factor of 2 . As in the case of classical Lamb waves, the fundamental mode is almost insensitive to the wafer thickness. The frequency control is then totally achieved by the poling period for that mode, offering attractive perspectives for the development of reliable high frequency devices.

In the first section of the paper, some design considerations are reported concerning the excitation of the Lamb modes, based on simple Green's function analyses. The fabrication process then is described, pointing out specific difficulties related to the use of $\mathrm{LiNbO}_{3}$. The second part of the paper is dedicated to the characterization of the devices and to theory/experiment assessment. The evolution of the spectral behavior of the device versus its thickness is theoretically and experimentally investigated and the mode shape and polarization are deduced from theoretical calculation. As a conclusion, the possible applications of devices based on PPTs on $\mathrm{LiNbO}_{3}$ are briefly discussed.

\section{DESIGN CONSIDERATIONS}

We first recall here the overall principle of the PPTs. It is fundamentally based on a periodically poled piezoelectric medium. Each side of this medium is metallized, providing a capacitive dipole in which elastic waves can be excited by phase construction. Such a periodically poled structure can be advantageously achieved on ferroelectric materials such as Zirconium - Titanium - Lead(Pb) (PZT) (Refs. 1 and 2) because of the rather small value of its coercive electric field 


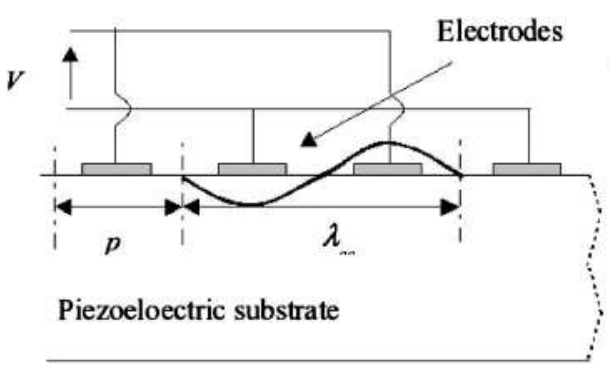

(a)

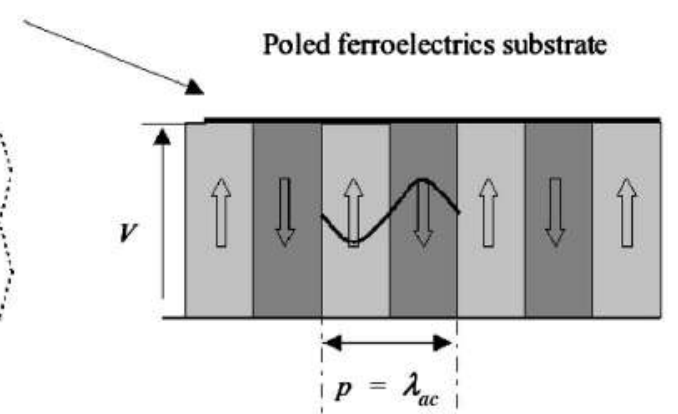

(b)
FIG. 1. (a) Comparison between principles of standard IDT-based devices and (b) poled ferroelectric film transducers. The polarity of the ferroelectric domains is indicated by gray levels and arrows the orientation of which corresponds to the direction of the $Z$ axis $(+Z, \uparrow ;-Z, \downarrow)$. (the absolute value of the electric field above which the ferroelectric poling can be controlled). The principal advantages of PPTs compared to standard periodic interdigital electrode transducers (IDTs) deposited atop piezoelectric materials are, first, the robustness of the excitation versus defects or surface contamination (no passivation is required to avoid short circuit as for standard IDTs) and, second, the opportunity to excite waves exhibiting a wavelength equal to the poling period, contrarily to standard IDTs for which the wavelength is equal to twice the mechanical period (Bragg condition). One can also note that comparably to film bulk acoustic resonators, ${ }^{8}$ it can operate with large excitation powers as the electrodes are separated by a bulk material layer. Figure 1 schematizes the general outlook of such a device and points out the benefits of the PPT compared to standard IDTs. As explained in Sec. I, the excitation and detection of guided elastic waves using PPTs have been demonstrated on an epitaxial PZT layer deposited on $\mathrm{SrTiO}_{3}{ }^{2}$ We inspect here the capability of the structure to excite elliptically polarized plate waves (Lamb modes) as the PPT's operation principle should be compatible which such an application.

In that purpose, the dispersion curves of Lamb modes on a $\mathrm{Z}$-cut $\mathrm{LiNbO}_{3}$ plate have been computed first along Green's function approach ${ }^{9}$ and are plotted in Fig. 2, giving a clear idea of the Lamb wave characteristics on such a substrate.

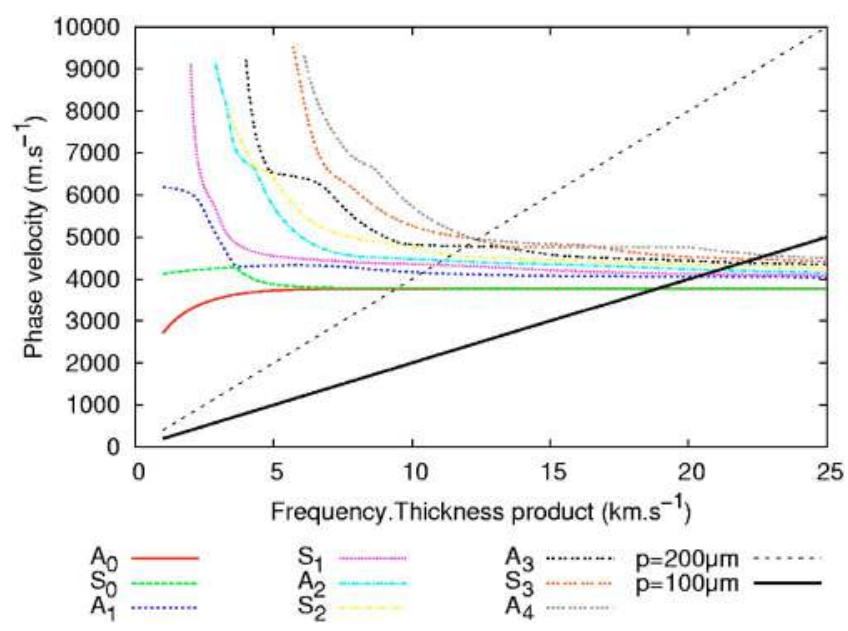

FIG. 2. (Color online) Dispersion curve of Lamb waves on a $Z$-cut $\mathrm{LiNbO}_{3}$ plate; frequency-thickness product ranges from 0 to $25 \mathrm{~km} \mathrm{~s}^{-1}$. The straight lines indicate the expected operating rules of the experimental PPT-based devices.
This allows us to point out the operating frequency of the excited modes for each sample configuration. A simple geometric construction gives the operating frequencies for each excited Lamb mode. ${ }^{10}$ For a given plate thickness $h$, the slope $m$ of a straight line passing through the origin is equal to the wavelength-plate thickness ratio $(m=\lambda / h)$. In our case, the thickness of the wafer is $500 \mu \mathrm{m}$, a standard value for commercially available material (e.g., Crystal Tech Inc.). We then made a trade-off between the difficulty to achieve very thin poled lines on thick wafers and the possibility to excite spectrally well separated modes. We consequently have considered periods equal to 200 and $100 \mu \mathrm{m}$, respectively, yielding values of $m$ equal to 0.4 and 0.2 (remember that in our devices, $\lambda=p$ ). Projecting the corresponding lines on the Lamb wave dispersion curves allows for the prediction of the wave velocity and hence of the operating frequency for each mode. Since the values of $m$ are always smaller than 1 (i.e., the frequency-thickness products corresponding to the test device are in the range of $5-50 \mathrm{~km} \mathrm{~s}^{-1}$ ), we could not take advantage of the high velocity of the $S_{0}$ mode. As a consequence, the first coupled mode on our device should always exhibit a wave velocity close to $3750 \mathrm{~m} \mathrm{~s}^{-1}$, yielding operating frequencies, respectively, equal to about 19 and $38 \mathrm{MHz}$. As shown further (Sec. IV), this first order prediction nicely meets the experimental values. Note that the corresponding coupling coefficients generally remain moderate, between $1 \%$ and $1 \%$. As explained above, practical implementation only should reveal the signature of symmetrical modes, assuming perfectly symmetrical transducer structures. The corresponding frequencies are compared to experimental values in Sec. IV.

\section{FABRICATION OF PPTS ON Z-CUT LINBO $_{3}$ PLATES}

As mentioned in Sec. I, the poling process can be rather easily applied to PZT for which the coercive field is small enough for an efficient control of the domain polarity. In the case of lithium niobate, this situation is quite different because of the large value of its coercive field $\left(21 \mathrm{kV} \mathrm{mm}^{-1}\right.$ compared to $5-10 \mathrm{kV} \mathrm{mm}^{-1}$ for PZT). As a consequence, the development of a dedicated poling bench was required to control the poling of thick $(500 \mu \mathrm{m}) \mathrm{Z}$-cut $\mathrm{LiNbO}_{3}$ plates. See, for instance, Ref. 11 for specific details on thick $\mathrm{LiNbO}_{3}$ $Z$-cut wafer poling process.

The poling bench mainly consists in a high voltage am- 


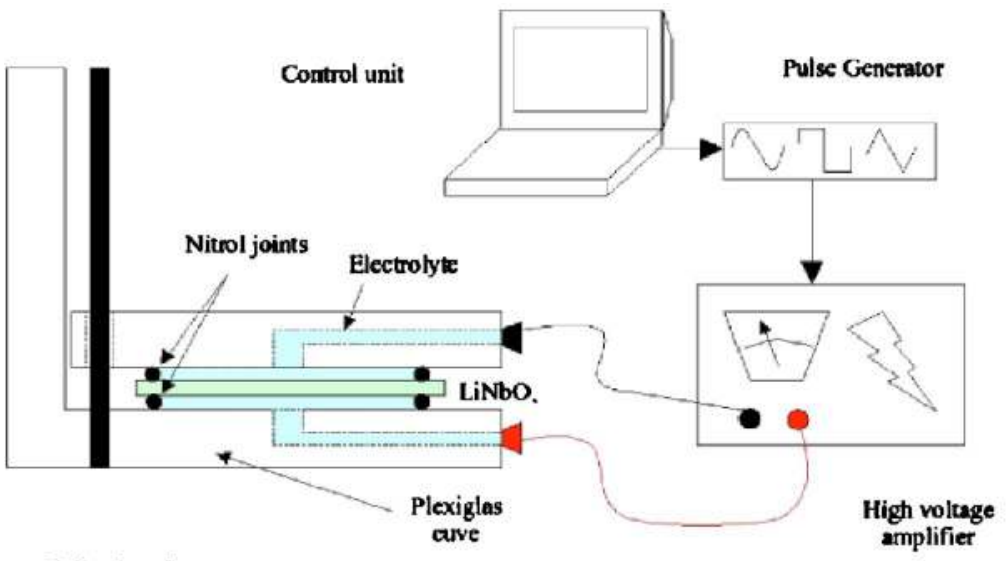

Poling bench

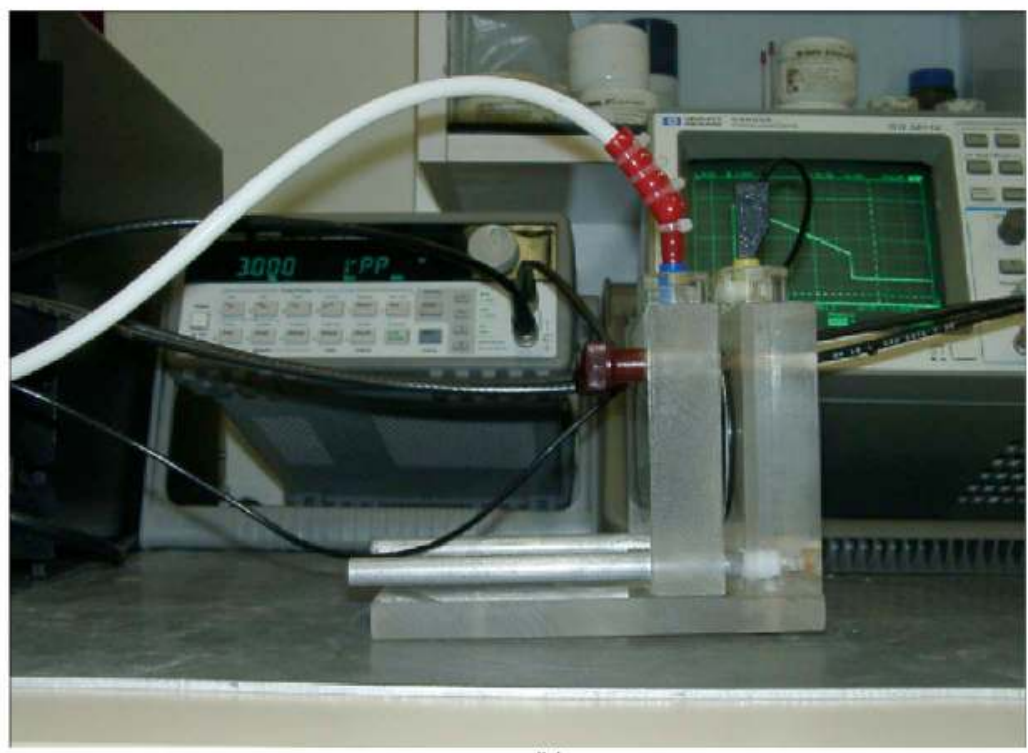

(b)
FIG. 3. (Color online) (a) Scheme of the ferroelectric plate poling bench and (b) photo of the experimental setup. plifier used to apply an electric field to the $\mathrm{LiNbO}_{3}$ wafer strong enough to invert the spontaneous polarization of the material [see Figs. 3(a) and 3(b)]. The domains to be poled are defined by a photoresist pattern on top of a plate surface. This yields a dielectric barrier strong enough to prevent any electric field to be located underneath the resist. The high poling voltage is applied to the plate following the sequence shown in Fig. 4 and using liquid electrodes made with a saturated lithium chloride solution. This sequence is designed to favor the domain nucleation, to stabilize the inverted domains (i.e., to avoid backswitching of the domains), and to avoid electrical breakdowns. ${ }^{11}$ The poling process is monitored by the measurement of the electric current crossing the wafer during the sequence. The signature of a successful domain inversion corresponds to the current discharge observed in Fig. 4. The poling can be easily

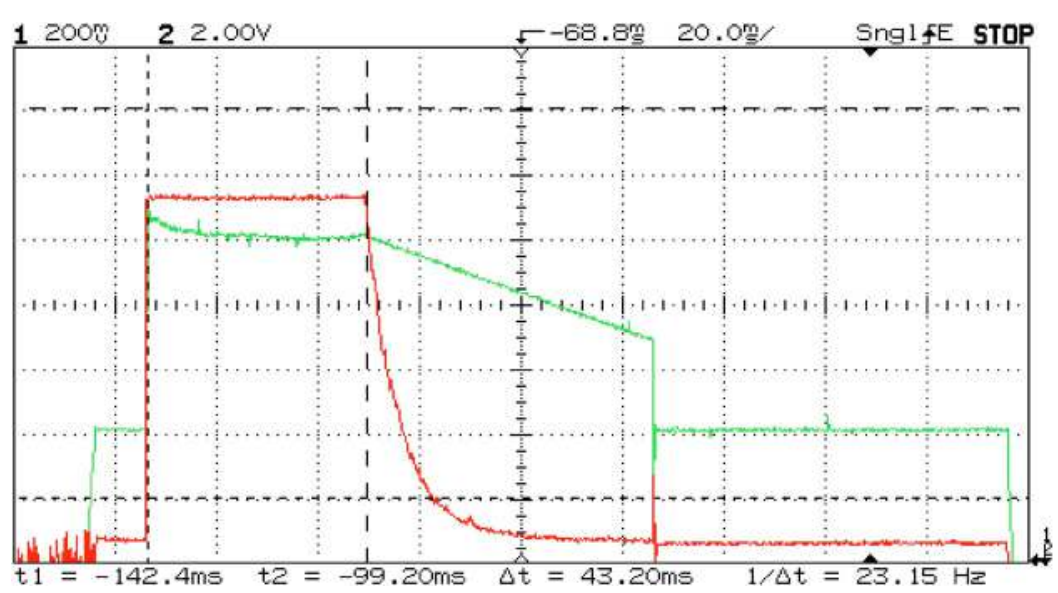

FIG. 4. (Color online) Domain inversion monitoring: probed high voltage applied on the wafer (green curve, poling sequence) and resulting crossing current (red curve). Voltage and current gain are, respectively, $1 \mathrm{~V} / 5000 \mathrm{~V}$ and $1 \mathrm{~V} / 5 \mathrm{~mA}$. First step: the applied electric field is smaller than the coercive limit to prepare the sample for the nucleation step. Second step: the applied field overcomes the coercive limit, yielding nucleation and consequently poling (this step lies between the two dashed cursors). Third step: linear decrease of the field to finish the nucleation (selftermination step, exponential decrease of the current). Fourth step: stabilization of the poling by applying a field smaller than the coercive limit (Ref. 11). 


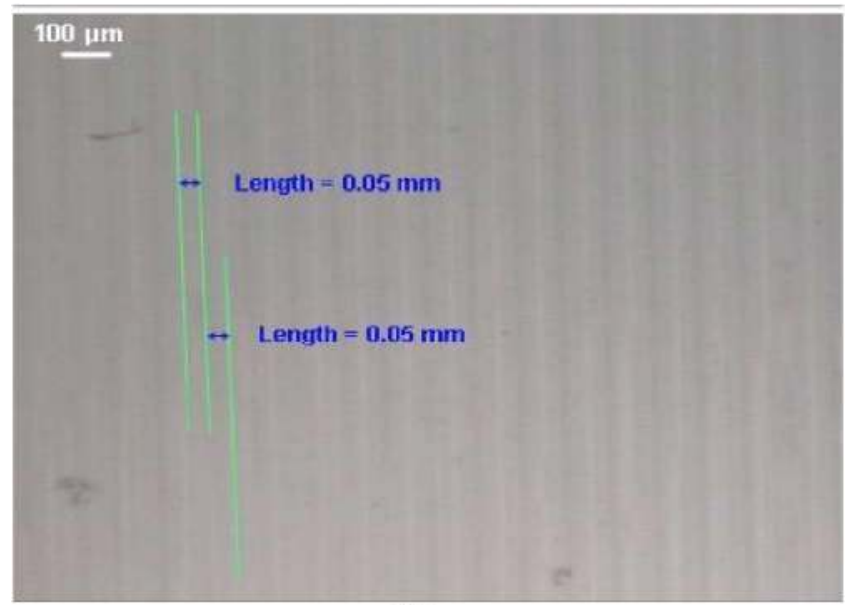

(a)

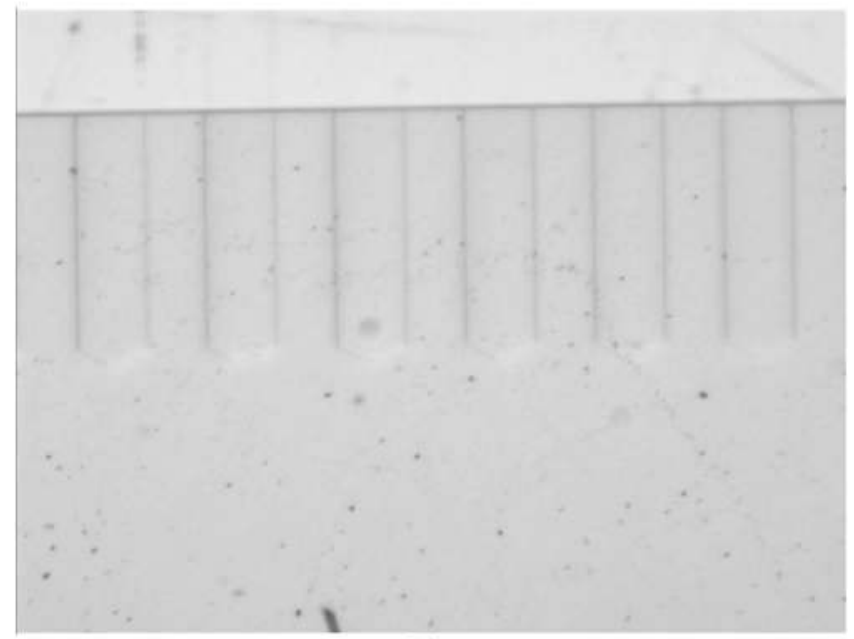

(b)

FIG. 5. (Color online) Optical checking of the poling efficiency: (a) In the case of a $100 \mu \mathrm{m}$ period device, evidence of contrast changes between $Z^{+}$ (native polarization), and $Z^{-}$(switched polarization) domains. (b) Evidence of contrast modification is in the vicinity of the poled domain walls at the poled strip edge (the whole plate is $Z^{+}$oriented, the poled strips are $Z^{-}$ oriented).

controlled by a simple optical postobservation, as it generates an optical contrast between and at the edge of the poled domains (see Fig. 5).

Figure 6 shows the implantation of the periodically poled structures on the wafer and of the top side electrodes

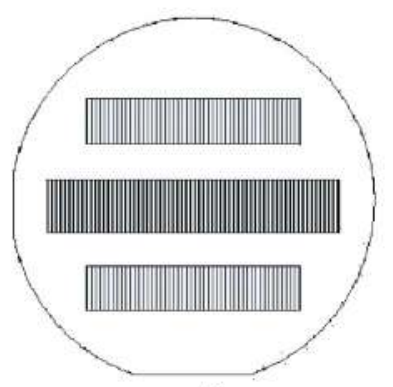

(a)

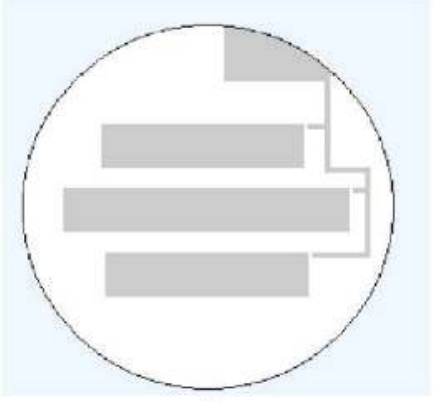

(b)
FIG. 6. (Color online) Implantation of the (a) periodically poled sequences and (b) top side electrode on the $Z$-cut $\mathrm{LiNbO}_{3}$ wafer (the bottom side is fully metallized).

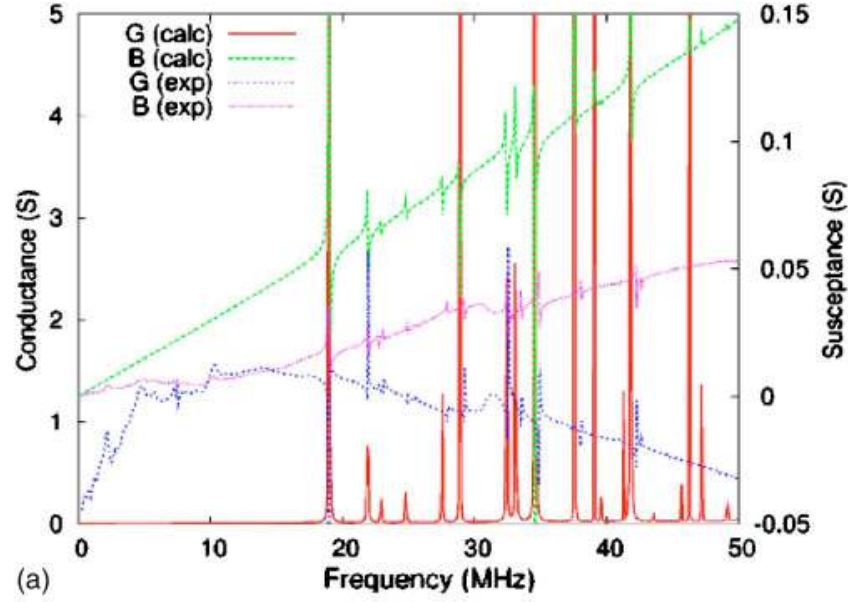

(a)

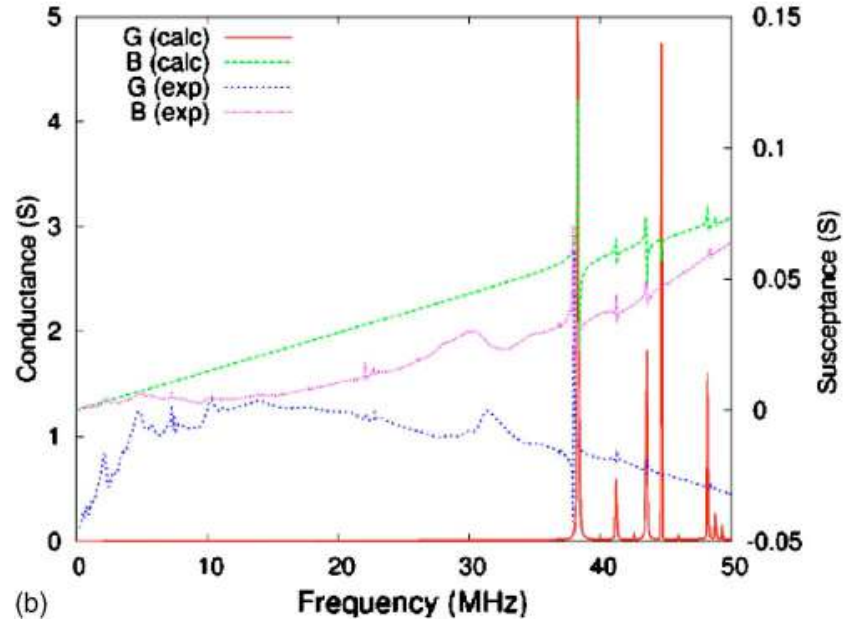

FIG. 7. (Color online) Comparison between theoretically predicted and experimental admittances for the two test vehicles for periods of (a) $p$ $=200 \mu \mathrm{m}$ and (b) $p=100 \mu \mathrm{m}$.

(the wafer back side is totally metallized). Note that the back side electrical contact is reported on the top surface by the metallization of the wafer sides (naturally induced by the sputtering process used to metallize the wafers).

The fabricated devices correspond to periodic transducers exhibiting periods $p$ equal to 200 and $100 \mu \mathrm{m}$, respectively, with a volume fraction ideally equal to $50 \%$. The achieved transducers exhibit 400 and 500 periods each, yielding quite long structures preventing the fabrication of more than three devices on a 3 in. wafer. The acoustic aperture was $10 \mathrm{~mm}$ for each device in order to avoid any diffraction effect. This kind of structure is assumed to only exhibit symmetrical modes, as there is no source of geomet-

TABLE I. Resonance frequencies of the $200 \mu \mathrm{m}$ period device.

\begin{tabular}{cccc}
\hline \hline Lamb modes & Green $(\mathrm{MHz})$ & FEA $(\mathrm{MHz})$ & Experiments $(\mathrm{MHz})$ \\
\hline$S_{0}$ & 18.9 & 19.0 & 18.92 \\
$A_{1}$ & 20.74 & $\ldots$ & $\ldots$ \\
$S_{1}$ & 21.66 & 21.9 & 21.9 \\
$A_{2}$ & 22.24 & $\ldots$ & $\ldots$ \\
$S_{2}$ & 22.86 & 22.9 & 23.11 \\
$A_{3}$ & 23.92 & $\ldots$ & $\ldots$ \\
$S_{3}$ & 24.66 & 24.79 & 24.9 \\
\hline
\end{tabular}


TABLE II. Resonance frequencies of the $100 \mu \mathrm{m}$ period device.

\begin{tabular}{cccc}
\hline \hline Lamb modes & Green $(\mathrm{MHz})$ & FEA $(\mathrm{MHz})$ & Experiments $(\mathrm{MHz})$ \\
\hline$S_{0}$ & 37.75 & 38.3 & 37.9 \\
$A_{1}$ & 40.52 & $\ldots$ & $\ldots$ \\
$S_{1}$ & 41.12 & 41.19 & 41.22 \\
$A_{2}$ & 42.12 & $\ldots$ & $\ldots$ \\
$S_{2}$ & 43.16 & 43.45 & 43.53 \\
$A_{3}$ & 43.88 & $\ldots$ & $\ldots$ \\
$S_{3}$ & 44.72 & 44.7 & 45.0 \\
\hline \hline
\end{tabular}

ric nor material-related asymmetries. It is expected to behave in a very close way to the one simulated by our own periodic finite element analysis (FEA) code which is devoted to the simulation of infinite periodic transducers. ${ }^{7}$ We also assume that edge effects should remain small enough to prevent major signal pollution, as there is no in-phase reflection effects on the wafer sides.

\section{EXPERIMENTAL RESULTS}

In this section, we present the electrical measurements of our PPT devices, obtained using a Süss Microtec radio frequency probing bench equipped with the so-called $Z$ probes connected to a Rohde \& Schwarz ZVRC network analyzer. The electrical admittances of the test devices have been measured and are compared to the theoretical harmonic admittances of the corresponding configurations. For the corresponding computations, finite elements based on second degree interpolation polynomials with four degrees of freedom were used (displacements along $x, y$, and $z$, and the electric potential) to obtain a high accuracy prediction. As it is shown further, this suggests that the excited modes may exhibit significant shear displacements contrarily to Lamb waves in isotropic media. These results are reported in Figs. 7 (a) and 7(b) for both considered devices. It shows that all the mode contributions to the electrical admittance are correctly predicted by the model. Tables I and II show the ac-

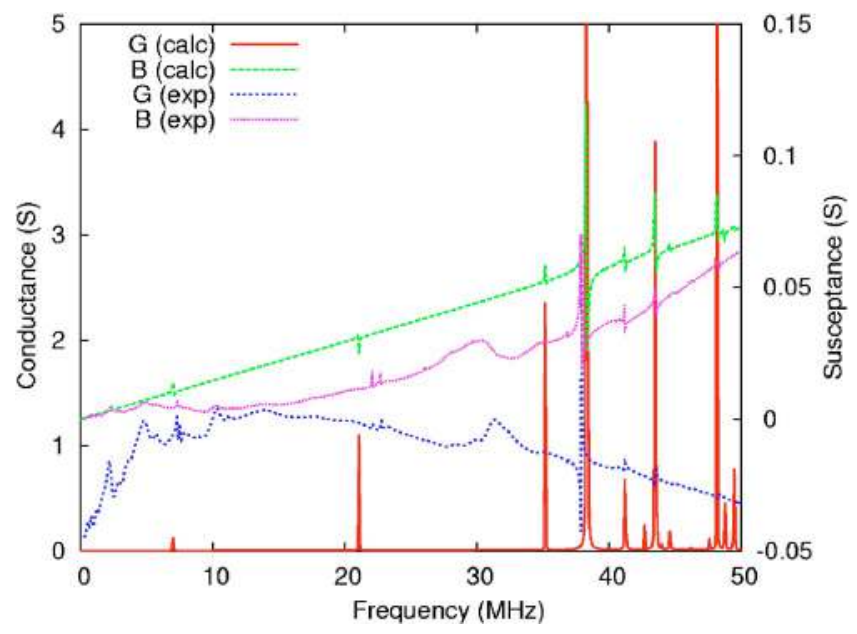

FIG. 8. (Color online) Influence of a volume fraction defect on the $100 \mu \mathrm{m}$ period transducer response ( $40 \% / 60 \%$ instead of $50 \%$ as expected). Spurious signals due to nonzero bulk wave excitation arise at low frequency (fundamental mode close to $7 \mathrm{MHz}$, third and fifth harmonics in the vicinity of 21 and $35 \mathrm{MHz}$, respectively).
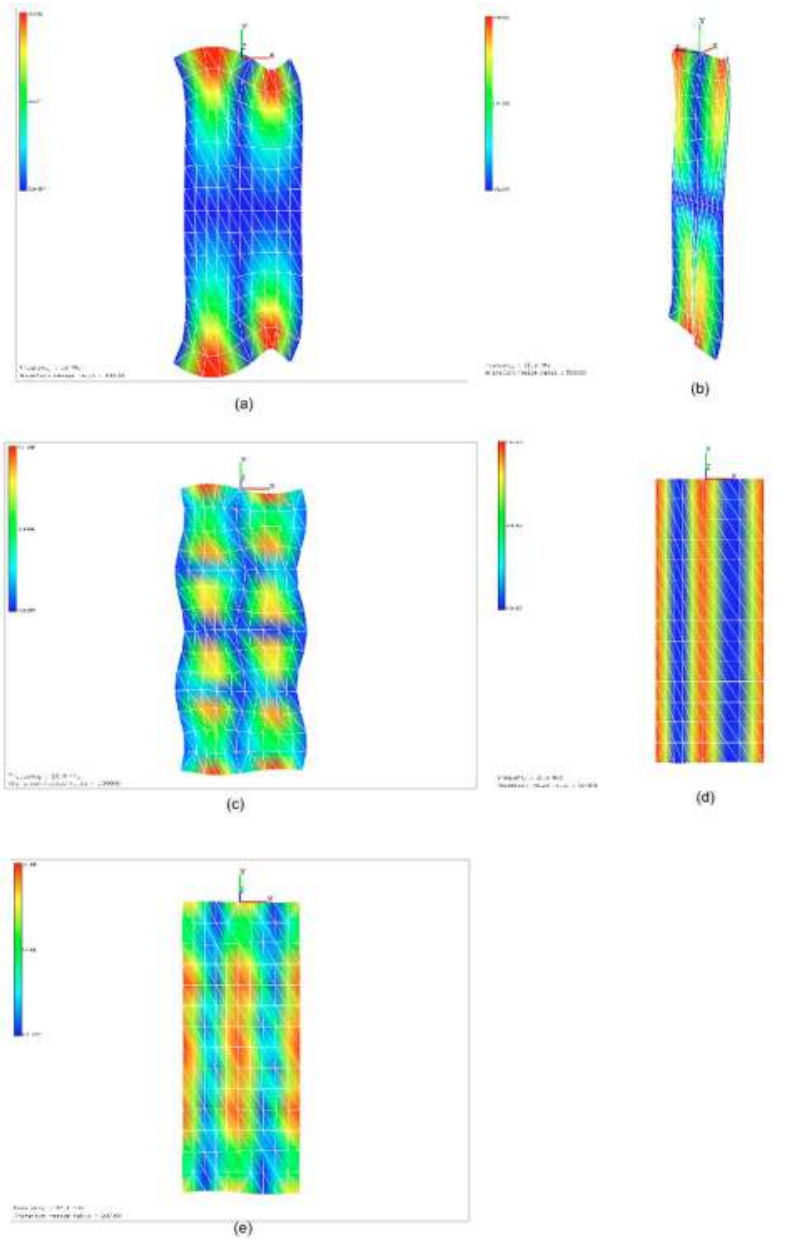

FIG. 9. (Color online) Shape of given modes of the $200 \mu \mathrm{m}$ period transducer [Fig. 5(b)]: (a) fundamental at $19 \mathrm{MHz}$, elliptically polarized; (b) mode 2 at $21.9 \mathrm{MHz}$, mainly shear polarization; (c) mode 6 at $28,9 \mathrm{MHz}$, high order elliptically polarized; (d) mode 7 at $31.8 \mathrm{MHz}$, pure longitudinal polarized; (e) mode 9 at $33.1 \mathrm{MHz}$, mainly longitudinal polarized (plus elliptical motion at the plate surfaces).

curacy of the predictions based on Green's function [Figs. 2(a) and 2(b)] and on the harmonic admittance. As explained above, antisymmetrical modes do not contribute to the transducer response since they do not comply with the excitation conditions.

We have related the contribution on the experimental curves near $7 \mathrm{MHz}$ of Fig. 7 to parasitic bulk waves due to the actual volume fraction of positively and negatively poled domains of the period. In the case the volume fraction would not rigorously equal 50\% (for instance, $45 \% \mathrm{Z}+155 \% \mathrm{Z}$ within the period), the longitudinal bulk wave and its odd harmonics [see the contribution at $21 \mathrm{MHz}$ in Fig. 7(b)] can be weakly excited. This was simulated for the $100 \mu \mathrm{m}$ period device and is reported in Fig. 8.

Thanks to the very good agreement between theory and experiments in Fig. 7, one can easily identify the shape of the modes of the different Lamb wave contributions, allowing to select one of those for its particular properties and polarization. Figures 9(a)-9(e) show some examples of modes excited in the plate for a $200 \mu \mathrm{m}$ period transducer. It can be seen on these graphs that elliptical, longitudinal, and generally polarized modes can be excited in the plate by our PPTs, 

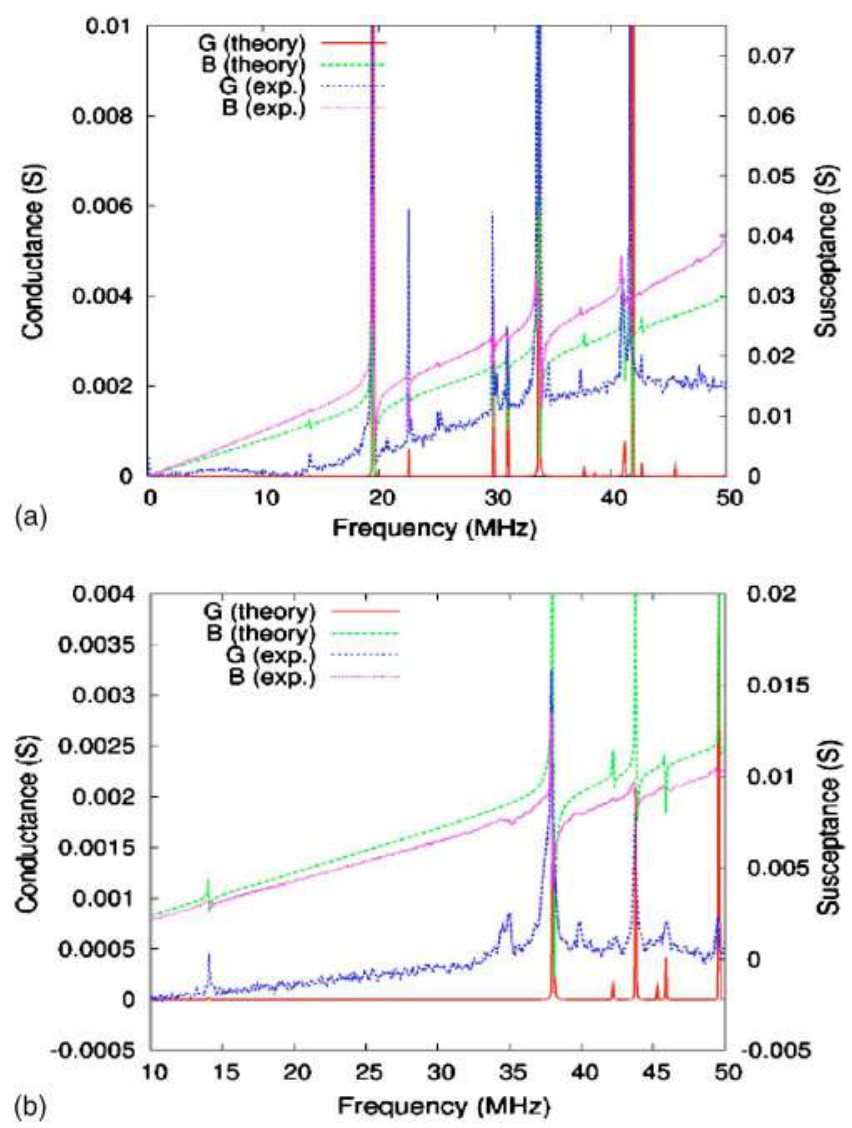

FIG. 10. (Color online) Theory/experiment assessment via electrical admittances after lapping the $\mathrm{LiNbO}_{3}$ plate down to $250 \mu \mathrm{m}$ for periods of (a) $p=200 \mu \mathrm{m}$ and (b) $p=100 \mu \mathrm{m}$.

as they do respect the symmetric boundary conditions of the device. We have reported in Tables I and II a comparison between experimental frequencies of the device admittance contribution with those predicted by the FEA model and those deduced by the analysis in Sec. II. The very good agreement between these different results is again emphasized, allowing for accurate design and analyses of such devices.

The last experimental work that has been achieved for this paper was to test the robustness of the polarization facing lapping and polishing techniques. These operations are required in order to fabricate $\mathrm{LiNbO}_{3} /$ silicon waveguides, as expected as a future development of our work. We consequently have lapped down the plate to a thickness of $250 \mu \mathrm{m}$ and then achieved new admittance measurements after polishing and metallization of the wafer. Again, the very nice theory/experiment agreement is met, as shown in Fig. 10, proving that the poling is strong enough to persist even after such treatments.

\section{CONCLUSION}

Periodically poled transducers have been successfully manufactured on $\mathrm{LiNbO}_{3} \mathrm{Z}$-cut plate, allowing for a very nice demonstration of the operation of periodically distributed piezoelectric domain transducers on such a material. Almost all the contributions to the electrical admittance of the two tested devices have been predicted by FEA, valuable for the mode shape identification. Elliptical, shear, and generally polarized waves are excited, demonstrating the capability of PPTs to excite any kind of plate waves. The poling process is found to be very resistant to lapping and polishing treatments, allowing for numerous kinds of process sequencing. This will be exploited for the fabrication of waveguides consisting of thinned $Z$-cut lithium niobate plates on silicon in a further development of this work. These waveguides could be used for sensor applications, but also for signal processing comparable to standard surface acoustic wave device achievements, provided the modes can be efficiently separated (i.e., the plate could be thin enough compared to the wavelength). Also acousto-optic devices could take advantage of such a transducer configuration to favor a strong interaction between optic and acoustic waves, provided the wavelength of both propagation can be properly matched.

\section{ACKNOWLEDGMENTS}

This work has been achieved in a collaborative project framework funded by the European Community as the InterReg project DOMINOS.

${ }^{1}$ S. Ballandras, W. Daniau, B. Gautier, D. Hauden, M. Wilm, V. Laude, V. Ruch, and S. Flambart, Proceedings of the Joint EFTF-IEEE International Frequency Control Symposium, Tampa Bay, FL, 2003 (unpublished), pp. 893-896.

${ }^{2}$ A. K. Sarin Kumar, P. Paruch, J.-M. Triscone, W. Daniau, S. Ballandras, L. Pellegrino, D. Marré, and T. Tybell, Appl. Phys. Lett. 85, 1757 (2004).

${ }^{3}$ M. Yamada, N. Nada, M. Saitoh, and K. Watanabe, Appl. Phys. Lett. 62, 435 (1993).

${ }^{4}$ M. H. Chou, J. Hauden, M. A. Arbore, and M. M. Fejer, Opt. Lett. 23, 1004 (1998).

${ }^{5}$ S. Ballandras, T. Pastureaud, V. Laude, W. Steichen, W. Daniau, R. Lardat, M. Solal, J. B. Briot, S. Chamaly, M. Doisy, X. Perois, and P. A. Girard, in Piezoelectric Materials for Macro/Macro-Systems, edited by D. Rémiens (Research Signpost, Besançon, 2003), pp. 183-197.

${ }^{6}$ A. Martinez, Ph.D. thesis, University of Franche-Comté, 2002.

${ }^{7}$ S. Ballandras, M. Wilm, P. F. Edoa, V. Laude, A. Soufyane, W. Steichen, and R. Lardat, J. Appl. Phys. 93, 702 (2003).

${ }^{8}$ R. Lanz and P. Muralt, IEEE Trans. Ultrason. Ferroelectr. Freq. Control 52, 946 (2005).

${ }^{9}$ A. Reinhardt, T. Pastureaud, S. Ballandras, and V. Laude, J. Appl. Phys. 94, 6923 (2003).

${ }^{10}$ S. Ballandras, A. Reinhardt, A. Khelif, M. Wilm, V. Laude, W. Daniau, and V. Blondeau-Pâtissier, J. Appl. Phys. 99, 054907 (2006).

${ }^{11}$ L. E. Myers, R. C. Eckardt, M. M. Fejer, R. L. Byer, W. R. Bosenberg, and J. W. Pierce, J. Opt. Soc. Am. B 12, 2102 (1995). 